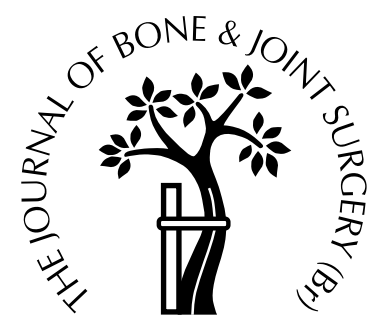

\title{
Tarsal tunnel syndrome caused by ganglia
}

\author{
M. Nagaoka, K. Satou
}

From Surugadai Nihon University Hospital, Tokyo, Japan

W e describe in 30 feet the occurrence of a tarsal tunnel syndrome caused by a ganglion. The presenting symptom was numbness or pain in the toes and the sole with paraesthesiae in the distribution of the medial plantar nerve in $63 \%$ of the patients. Swellings which were not palpable were detected by ultrasonography. Twenty-nine patients were treated by operation. Most ganglia originated from the talocalcaneal joint, and five were associated with a talocalcaneal coalition. The surgical outcome was satisfactory in all patients except one who had a further operation for a recurrence of the ganglion.

J Bone Joint Surg [Br] 1999;81-B:607-10.

Received 12 October 1998; Accepted after revision 4 November 1998

Tarsal tunnel syndrome was first described by $\mathrm{Keck}^{1}$ and $\mathrm{Lam}^{2}$ in 1962. The aetiology has been considered to be idiopathic or post-traumatic, ${ }^{3-9}$ with some cases due to a space-occupying lesion. ${ }^{10-14}$ There are a number of reports which describe a tarsal tunnel syndrome caused by a ganglion, of which only a few give an adequate number of cases. $4,7,15-24$

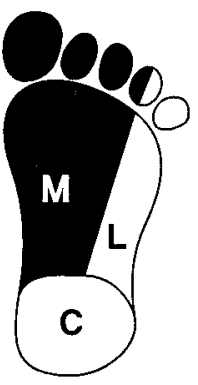

19 feet

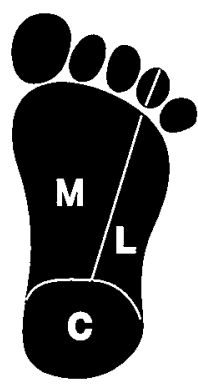

6 feet

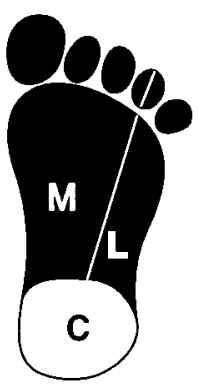

3 feet

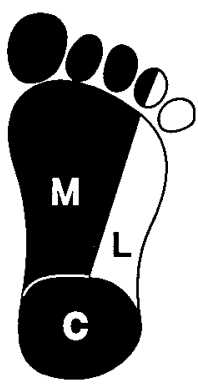

2 feet
We have reviewed 30 patients with a tarsal tunnel syndrome diagnosed and treated in our department, and have analysed the clinical presentation, diagnosis, and the outcome of surgery.

\section{Patients and Methods}

Between January 1978 and December 1997 we treated 30 patients (30 feet) for tarsal tunnel syndrome caused by ganglia. There were 16 men and 14 women, with a mean age of 40.5 years (12 to 70). The right foot was affected in 16 patients and the left in 14 .

The syndrome presented with numbness or pain in the toes and sole in 29 patients (97\%) and pain in $17(57 \%)$. Only two complained of a burning pain in the foot. Hypoaesthesia was found in 25 patients (83\%). Paraesthesiae and pain were localised to the distribution of the medial plantar nerve in 19 patients, over the distribution of both medial and lateral plantar nerves in three, over the distribution of the medial plantar nerve and the medial calcaneal branch in two, and involved the whole plantar aspect innervated by both medial and lateral plantar nerves as well as the medial calcaneal branch in six (Fig. 1). Thus
M. Nagaoka, MD, Assistant Professor

K. Satou, MD, Professor

Department of Orthopaedics, Surugadai Nihon University Hospital, 1-8-13

Kanda Surugadai, Chiyoda-ku, Tokyo 101, Japan.

Correspondence should be sent to Dr M. Nagaoka.

(C)1999 British Editorial Society of Bone and Joint Surgery 0301-620X/99/49546\$2.00

all patients had paraesthesiae in the distribution of the medial plantar nerve. A positive Tinel sign was elicited by percussion in the tarsal tunnel in all affected feet. The abductor hallucis muscle was atrophic in eight patients (27\%); only a few complained of weakness of the foot. A mass was palpable below the medial malleolus of the tibia in 28 patients, but not in the remaining two. 


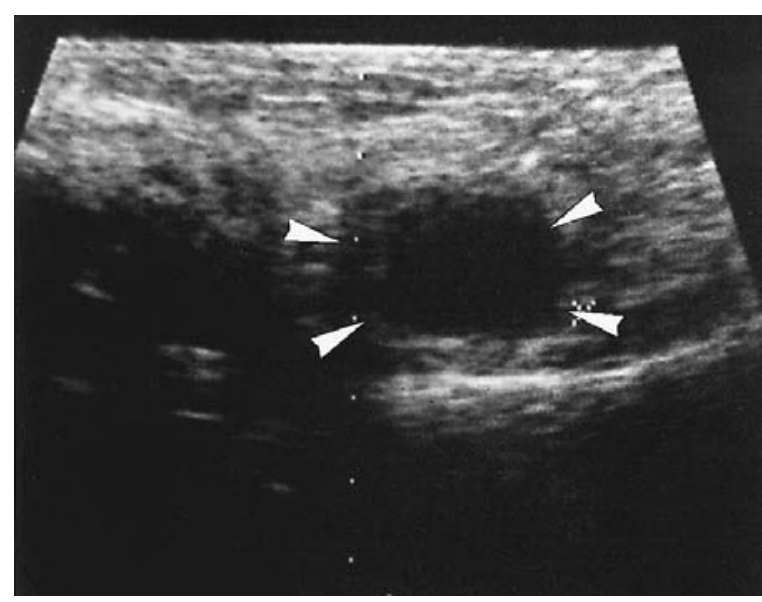

Fig. 2

Ultrasonogram of a 51-year-old man showing a round hypoechoic area (arrowheads) in the tarsal tunnel.

A mechanical sector scanner containing a $10 \mathrm{MHz}$ ultrasound transducer and an Aloka SSD650CL echograph were used to examine 12 feet by short- and long-axis crosssectional ultrasonography. The ganglion was depicted as an echo-free or hypoechoic area in all affected feet. Some of the ganglia were round, but others were multilobular (Figs 2 and 3); some of those associated with talocalcaneal coalitions were gourd-shaped and found on the bridge of the coalition (Fig. 4). Ultrasonography was very useful in planning surgical management and in two patients the presence of two separate ganglia, which were not palpable, was demonstrated by preoperative ultrasonography.

The results of operation were evaluated on a four-point scale; excellent (normal, without numbness, with or without persistent slight hypoaesthesia and cold intolerance), good (the presence of hypoaesthesia, not causing discomfort during daily activities, without numbness or pain), fair (persistence of hypoaesthesia which produces discomfort in daily life and pain in the standing position or during walking, despite symptomatic improvement) and poor (lack of symptomatic improvement or aggravation compared with the preoperative condition).

\section{Results}

Electrodiagnostic studies were carried out on 26 feet. In 17 the action potentials of the tibial sensory nerve were absent between the big toe and the area proximal to the tarsal tunnel. The conduction velocity of sensory nerves was reduced in six feet. The distal motor latencies, measured from the abductor hallucis muscle, were prolonged in 11 feet, although the compound muscle action potential was recorded in all affected feet studied.

The ganglion disappeared spontaneously in one patient. The remaining 29 were treated surgically. At operation, the ganglia were identified as swellings of various forms and shapes. Some lifted or encircled the tibial nerve while

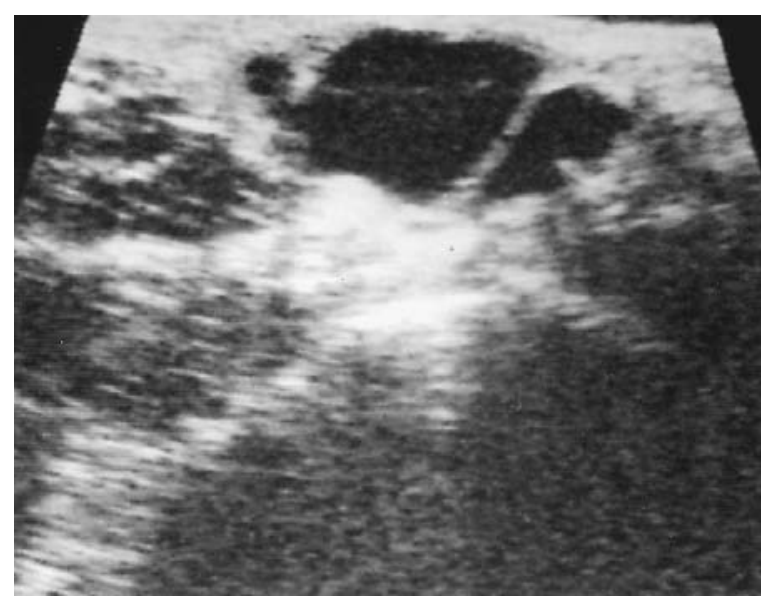

Fig. 3

Ultrasonogram of a 43-year-old woman showing a multilobular hypoechoic area in the tarsal tunnel.

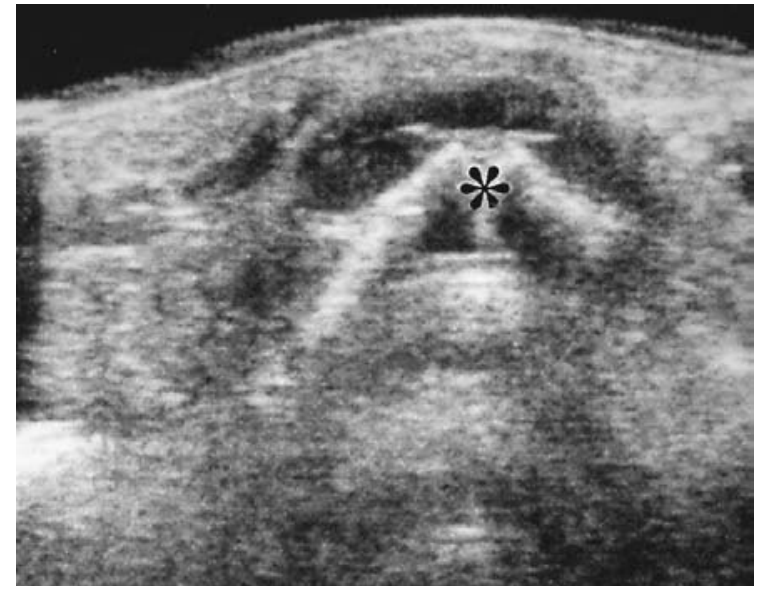

Fig. 4

Ultrasonogram of a 35-year-old man showing a hypoechoic area over the beak-shaped talocalcaneal coalition (asterisk).

others moved on to the tibial nerve when the ankle was dorsiflexed. All ganglia compressed the medial plantar branch of the posterior tibial nerve and large lesions compressed the tibial nerve more proximally. In 15 feet the ganglion originated from the talocalcaneal joint in 14 and the talocrural joint in one. In the remaining 15 the origin could not be identified.

The ganglion was excised in 25 feet; five of the lesions were large and adhered to the adjacent nerves, resulting in incomplete excision. Four were treated by drainage alone. The cause of the tarsal tunnel syndrome was a ganglion alone in 23 feet, a ganglion associated with a talocalcaneal coalition in five, and a ganglion associated with varicosities in one. Postoperative follow-up ranged from eight to 114 months (mean, 27.5). Of the 29 feet treated surgically, numbness and pain disappeared immediately after operation in six and, in the others, pain was relieved within three months. A revision operation was carried out on one 


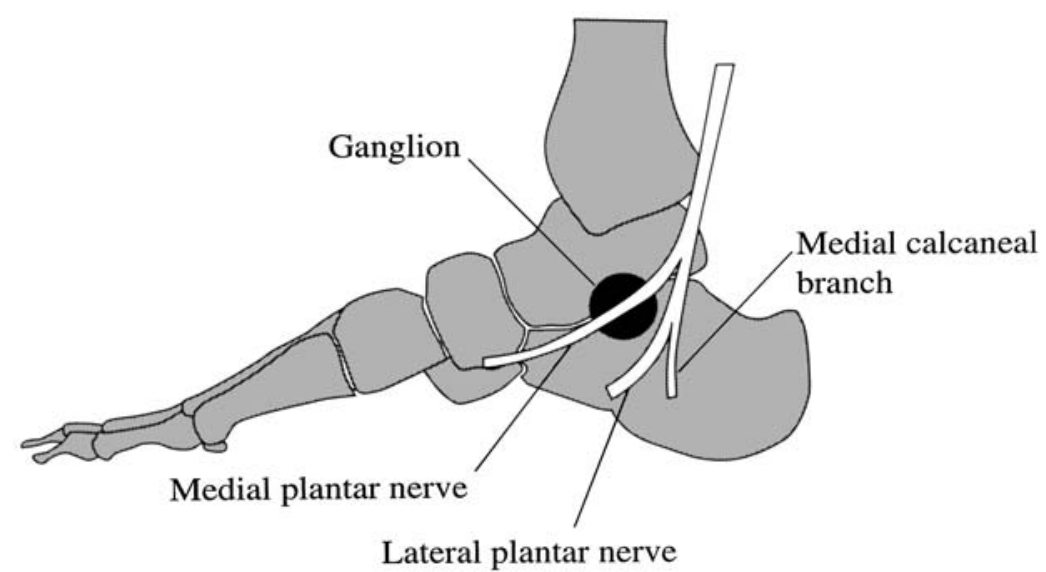

Fig. 5

Most patients presented with sensory disturbances limited to the distribution of the medial plantar nerve. This reflects the high prevalence of ganglia arising from the talocalcaneal joint.

patient, a 44-year-old woman, in whom the ganglion was associated with a talocalcaneal coalition. The ganglion compressed the medial plantar nerve and was excised during the first operation, leaving the coalition untreated. Ten months later, the ganglion recurred and produced numbness. The patient had a second procedure to treat the coalition, as well as the recurrent ganglion. Other associated ganglia were resected together with the coalition at the same operation. Ganglia recurred in four other patients at sites other than the original but caused no neurological symptoms.

Overall, the surgical outcome was excellent in 21 feet and good in eight. The average vertical and horizontal diameters of the ganglia measured in 20 feet were $11.9 \pm 5.3$ and $16.6 \pm 7.9 \mathrm{~mm}$, respectively. The surgical outcome did not correlate with the size of the lesion nor was there any association with the electrophysiological findings.

\section{Discussion}

Various space-occupying lesions can cause a tarsal tunnel syndrome. In addition to ganglia and talocalcaneal coalition, it has been described in association with neurofibroma, an intraneural ganglion, the flexor digitorum accessorius longus muscle, or a lipoma. ${ }^{4,6,10-14}$

The clinical manifestations of tarsal tunnel syndrome are diagnostic. $^{3,20}$ A careful physical examination will demonstrate the presence of a palpable or diffuse swelling in the tarsal tunnel. Aspiration may be useful for the diagnosis of a ganglion. Puncture is not recommended because branches of the tibial nerve in the tarsal tunnel may be injured, particularly since they may cross over the swelling. In one of our cases, the diagnosis was made by puncture; pain in the plantar aspect was alleviated but intense irritation persisted at the site of the puncture. Imaging is a better method of diagnosis and we found ultrasonography to be useful. Since ganglia are depicted as hypoechoic or echo-free areas, they can be identified easily and the site, form and size assessed. Some occult ganglia which are not palpable can be detected by ultrasonography. Many ganglia are associated with a talocalcaneal coalition, and we recommend that diagnosis should not only be based on radiography, but also on ultrasonography.

Paraesthesiae developed along the distribution of nerves compressed in the tarsal tunnel. In this region, the posterior tibial nerve gives rise to three branches. ${ }^{26}$ They are the medial and lateral plantar nerves, and the medial calcaneal branch. If the ganglion is located proximal to the bifurcation of the tibial nerve, paraesthesiae will be present in the whole plantar aspect of the foot. When the distal part of the medial plantar nerve is compressed, numbness develops only in the medial aspect of the sole near the big toe. Of the 30 patients reviewed in our study, 19 had sensory disturbances limited to the medial distribution of the plantar nerve reflecting the high prevalence of ganglia arising from the talocalcaneal joint (Fig. 5).

Operation is usually considered to be more successful in the treatment of tarsal tunnel syndrome caused by a spaceoccupying rather than other lesions. ${ }^{20,23}$ Our results were satisfactory. Recurrence was not associated with neurological symptoms and was attributed to incomplete excision of large ganglia.

No benefits in any form have been received or will be received from a commercial party related directly or indirectly to the subject of this article.

\section{References}

1. Keck C. The tarsal-tunnel syndrome. J Bone Joint Surg [Am] 1962; 44-A:180-2.

2. Lam SJS. A tarsal-tunnel syndrome. Lancet 1962;2:1354-5.

3. Bailie DS, Kelikian AS. Tarsal tunnel syndrome: diagnosis, surgical technique, and functional outcome. Foot Ankle Int 1998;19:65-72.

4. Distefano V, Sack JT, Whittaker R, Nixon JE. Tarsal tunnel syndrome: review of literature and two case reports. Clin Orthop 1972;88:76-9.

5. Edwards WG, Lincoln CR, Bassett FH III, Goldner JL. The tarsal tunnel syndrome: diagnosis and treatment. JAMA 1969;207:716-20.

6. Cimino WR. Tarsal tunnel syndrome: review of the literature. Foot Ankle 1990;11:47-52.

7. Mann RA. Tarsal tunnel syndrome. Orthop Clin N Am 1974;5:109-15.

8. Radin EL. Tarsal tunnel syndrome. Clin Orthop 1983;181:167-70.

9. Stern DS, Joyce MT. Tarsal tunnel syndrome: a review of 15 surgical procedures. J Foot Surg 1989;28:290-4.

10. Aydin AT, Karaveli S, Tuzuner S. Tarsal tunnel syndrome secondary to neurilemmoma of the medial plantar nerve. J Foot Surg 1991; 30:114-6. 
11. Belding RH. Neurilemmoma of the lateral plantar nerve producing tarsal tunnel syndrome: a case report. Foot Ankle 1993;14:289-91.

12. Menon J, Dorfman HD, Renbaum J, Friedler S. Tarsal tunnel syndrome secondary to neurilemmoma of the medial plantar nerve: a case report and review of the literature. J Bone Joint Surg [Am] 1980;62-A:301-3.

13. Myerson M, Soffer S. Lipoma as an etiology of tarsal tunnel syndrome: a report of two cases. Foot Ankle 1989;10:176-9.

14. Sammarco GJ, Stephens MM. Tarsal tunnel syndrome caused by the flexor digitorum accessorius longus: a case report. J Bone Joint Surg [Am] 1990;72-A:453-4.

15. Brooks DM. Nerve compression by simple ganglia: a review of thirteen collected cases. J Bone Joint Surg [Br] 1952;34-B:391-400.

16. Brown RJ. Tarsal tunnel syndrome due to a ganglion: a case report. Ulster Med J 1982;51:127-9.

17. Kenzora JE, Lenet MD, Sherman M. Synovial cyst of the ankle joint as a cause of tarsal tunnel syndrome. Foot Ankle 1982;3:181-3.

18. Macfarlane IJ, Du Toit SN. A ganglion causing tarsal tunnel syndrome. S Afr Med J 1974;481:2568.
19. Matricali B. Tarsal tunnel syndrome caused by ganglion compression. J Neurosurg Sci 1980;24:183-5.

20. Pfeiffer WH, Cracchiolo M III. Clinical results after tarsal tunnel decompression. J Bone Joint Surg [Am] 1994;76-A:1222-30.

21. Pho RW, Rasjid C. A ganglion causing the tarsal tunnel syndrome: report of a case. Aust NZ J Surg 1978;48:96-8.

22. Taguchi Y, Nosaka K, Yasuda K, et al. The tarsal tunnel syndrome: report of two cases of unusual cause. Clin Orthop 1987;217:247-52.

23. Takakura Y, Kitada C, Sugimoto K, Tanaka Y, Tamai S. Tarsal tunnel syndrome: causes and results of operative treatment. $J$ Bone Joint Surg [Br] 1991;73-B:125-8.

24. Yamamoto S, Tominaga Y, Yura S, Tada H. Tarsel tunnel syndrome with double causes (ganglion, tarsal coalition) evoked by ski boots: case report. J Sports Med Phys Fitness 1995;35:143-5.

25. Takakura Y, Kumai T, Takaoka T, Tamai S. Tarsal tunnel syndrome caused by coalition associated with a ganglion. J Bone Joint Surg [Br] 1998;80-B:130-3.

26. Dellon AL, Mackinnon SE. Tibial nerve branching in the tarsal tunnel. Arch Neurol 1984;41:645-6. 